\title{
Lipids contribute to epigenetic control via chromatin structure and functions
}

\author{
Renad Zhdanov ${ }^{1}$, Eric C. Schirmer ${ }^{2}$, Anuroop V. Venkatasubramani ${ }^{3,4}$, Alastair R. W. Kerr ${ }^{2}$, Elena Mandrou ${ }^{3}$, \\ Giovanny Rodriguez-Blanco ${ }^{3}$, and Alexander Kagansky*3 \\ ${ }^{1}$ Institute of Fundamental Medicine and Biology, Kazan Federal University, Kazan 420008 and Russian Institute for \\ Advanced Study at Moscow Pedagogical State University, Moscow 119571 Russian Federation \\ ${ }^{2}$ Wellcome Trust Centre for Cell Biology, University of Edinburgh, Kings Buildings, Michael Swann Bldg., Max Born Crescent, \\ Edinburgh EH9 3BF, UK \\ ${ }^{3}$ MRC Human Genetics Unit, Institute of Genetics and Molecular Medicine, University of Edinburgh, Western General \\ Hospital, Crewe Road, Edinburgh EH4 2XU, UK \\ ${ }^{4}$ Department of Biosciences, P.O. Box 65 (Viikinkaari 1), 00014, University of Helsinki, Helsinki, Finland \\ *Corresponding author's e-mail address: kagasha@yahoo.com
}

Published online: 6 October 2015 (version 1); 23 August 2016 (version 2)

Cite as: Zhdanov R, Schirmer EC, Venkatasubramani AV, et al. (2016). Lipids contribute to epigenetic control via chromatin structure and functions. ScienceOpen Research, 10(15), (DOI: 10.14293/S2199-1006.1.SOR-LIFE.AUXYTR.v2)

Reviewing status: The review of this article was author-mediated. The ScienceOpen editorial office has verified and confirmed the identities of the reviewers. The endorsement statement and reviewers names are provided below. The article is also available for continuous post-publication peer review on its dedicated ScienceOpen homepage.

\section{Peer Review by Endorsement Statement:}

"I have read this article, given feedback to the authors and now feel that it is of appropriate quality to be included in the scientific literature and be part of the open scientific discourse."

Nick Barlev, PhD

Associate Professor of Biochemistry

Department of Biochemistry

University of Leicester
Vasily Ogryzko

Director of Research, 2nd grade

UMR 8126 Signalisation,

noyaux et innovations en oncologie, CRNS

Primary discipline: Life sciences

Secondary discipline: Cell biology, Cancer biology, Molecular biology, Genetics

Keywords: acylation, Epigenetics, lipid, cancer, chromatin, epigenetic code, histone, metaboloepigenetics, gene expression, lipidome

\section{ABSTRACT}

Isolated cases of experimental evidence over the last few decades have shown that, where specifically tested, both prokaryotes and eukaryotes have specific lipid species bound to nucleoproteins of the genome. In vitro, some of these lipid species exhibit stoichiometric association with DNA polynucleotides with differential affinities toward certain secondary and tertiary structures. Hydrophobic interactions with inner nuclear membrane could provide attractive anchor points for lipid-modified nucleoproteins in organizing the dynamic genome and accordingly there are precedents for covalent bonds between lipids and core histones and, under certain conditions, even DNA. Advances in biophysics, functional genomics, and proteomics in recent years brought about the first sparks of light that promises to uncover some coherent new level of the epigenetic code governed by certain types of lipid-lipid, DNA-lipid, and DNA-protein-lipid interactions among other biochemical lipid transactions in the nucleus. Here, we review some of the older and more recent findings and speculate on how critical nuclear lipid transactions are for individual cells, tissues, and organisms.

\section{INTRODUCTION}

The importance of many lipid classes in biology is without question as they act as secondary messengers and signaling molecules that are added post-translationally to proteins to regulate their function and targeting. Species of a variety of lipid classes (e.g., phospholipids, sphingolipids, neutral lipids, cholesterol, fatty acids) are also responsible for the formation of membrane barriers between cellular compartments. Membrane lipids hold a unique place among cellular biomolecules for their dual nature: they have both hydrophobic long carbon side chains (up to 28 carbon atoms chain length, presumably of trans-configuration and rarely hydroxylated) and hydrophilic polar head groups (conjugated to hydrophobic part by esterand/or ether bond) that enable them to interact with both organic and aqueous environments. To form lipid bilayer membranes, the hydrophobic regions interact with one another while their polar side chains on the outside interact with the aqueous cellular environment. At the same time, a variety of lipid classes at the edge of the cellular compartment provide a stably organized hydrophobic environment to support functions 
such as assembly and transport of various lipid signaling molecules, lipid-modified proteins, and membrane proteins. Moreover, the interplay between lipid species and proteins has been shown to alter the structural properties of proteins [1] and this has significant ramifications for human diseases such as Alzheimer's, Parkinson's, and prion diseases [2]. Both membrane lipids and lipid species lacking strong polar groups serve as signaling molecules in apoptosis [3], in lymphocyte activation [4], in membrane targeting inhibition [5], and systemic functions in organisms ranging from inflammation to cancer. Functions of lipid species as energy storage molecules are well known (e.g. triglycerides) and yet they also serve a wide range of essential functions, such as the source of fat-soluble vitamins and metabolic regulators. Unsurprisingly, lipid metabolism is critical for cell fate as well as for health and disease, being involved in respiratory and renal pathologies, in obesity, in neurological disorders, and finally in a wide range of cardiovascular diseases and cancer [6]. Part of what makes lipid species of different classes such an effective cellular component is their enormous diversity. There are just four nucleotides that code for the entire human genome and 21 amino acids that code for proteins. On the other hand, there are more than thousands of distinct lipid types in any given cell. This is possible because of the building block nature, even within each individual lipid. Each additional carbon atom in a lipid species can change its properties, and typical cellular lipid species range from 4 to 28 carbons. Similarly, each trans-double bond added within the carbon chain of lipid species further alters its properties. For example, many microorganisms change their lipid membrane profile with changing temperatures or pressure (such as deep ocean organisms) to increase the number of double bonds in their side chains, thus rendering the membranes more rigid $[7,8]$. Moreover, the more complex lipid classes have multiple carbon chains in addition to a variety of polar head groups, which could essentially lead to an infinite number of possibilities. Lipidomics aims to identify and characterize the lipid species and classes of the cell and to further investigate their structure and functions. Lipidomes of various organisms such as fruit fly have been recently reported [9], along with the fulllipidome profiling of yeast [10], blood plasma [11], viruses $[12,13]$, and mammalian epithelial cell lines [14,15]. Whereas the lipid composition of Drosophila melanogaster and Homo sapiens are strikingly alike, in other cases, the abundance of a particular lipid species compared to another is directly reversed (see Figure 1). It is reasonable to wonder whether the unchanging lipid species perform an indispensable function. On the other hand, it is not always clear whether glaring differences such as the enrichment of phosphatidylinositol (PI) in Saccharomyces cerevisiae plasma and endosomal membranes [16], indicate unique functions for specific needs of different organisms or if these dissimilarities simply reflect divergent evolution.
Although large-scale projects such as Lipid Maps and Eurolipidomics have resulted in significant progress to this field, very little is known about the function of lipid species inside the nucleus [17]. Phospholipids (PLs), ceramides (Cer), and sphingosines were found to impact on replication and transcription in both prokaryotes and eukaryotes [18]. For example, gene expression was altered upon treatment of the cells with gamma-linoleic acid [19]. Negatively charged PL species such as PI, cardiolipin (CL), phosphatidylserine (PS), and phosphatidylglycerol (PG) stimulate RNA synthesis, while phosphatidylcholine (PC), phosphatidylethanolamine (PE), and sphingomyelin (SM) inhibit this process [20]. Although the extremely complex lipid environment within a cell often makes it difficult to distinguish direct from indirect effects, clear lipid effects on nuclear functions have also been shown in fully controlled in vitro experiments. For example, a recent study indicated that a particular sphingolipid species could modulate DNA polymerase activity [21].

There are many plausible hypothetical mechanisms for nuclear lipid functions beyond membrane compartmentalization and signaling. Lipid species could directly affect DNA supercoiling, which is important for transcription, replication, and recombination/repair. Consistent with this hypothesis, selected natural and synthetic lipids inhibit the functioning of prokaryotic topoisomerase and polymerase enzymes via yet unidentified mechanisms [22-24]. Here, we will review some of the nuclear lipid species and discuss their known and/or possible functions. In particular, we will describe experiments that led us to hypothesize that lipid species may constitute a significant informational layer in the gene regulation space, conceptually partial to, what previously has been referred to as, "epigenetic code." While we are only starting to isolate lipid moieties serving as structural constituents of chromatin, it is increasingly clear that the involvement of lipids in gene regulation is manifold. While emerging/future technologies, such as lipid labeling, lipid-protein and lipid-DNA crosslinking, and super-resolution microscopy, warrant exciting big data on molecular mechanisms that cells employ to engage lipid species in order to regulate epigenetic processes, we will only briefly touch upon cases where certain lipid classes could be placed on the map, for playing roles in gene expression and chromosome dynamics. We will first look at the nuclear envelope, get through to its inner side, then briefly look inside the nucleoplasm, at the chromatin, the "genome wrap," and in conclusion, at the metabolo-epigenetic transactions, dynamically engaging nuclear proteins (including histones) on one side, and lipid species on the other.

\section{THE LIPID ENVIRONMENT OF THE NUCLEAR ENVELOPE}

The various cell membranes differ in lipid composition. For instance, PC is the most abundant lipid species in cell membranes of many species, while sphingolipids are next ones on the list being observed in the plasma membrane, while they are almost undetectable in the endoplasmic reticulum (ER), where PE and PI considerably contribute [16]. It has also been observed that the lipid composition change between the ER and Golgi complexes [16]. Indeed, the lipid composition of each cellular organelle has 


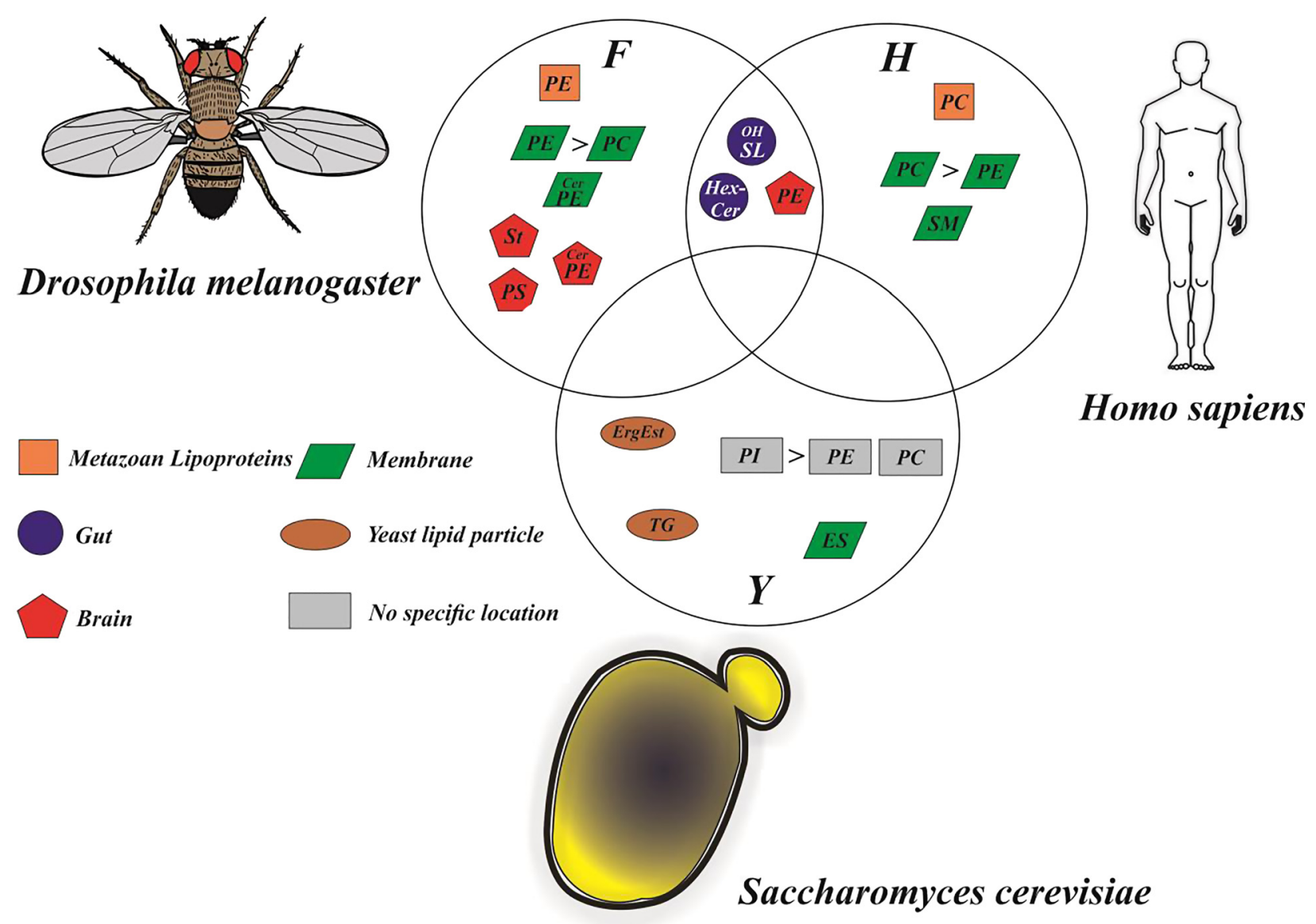

Figure 1. Eukaryotic lipidomes compared. Shotgun analysis of total lipids from fruit fly ( $F$ ), budding yeast $(\mathrm{Y})$, and human cell lines $(\mathrm{H})$ allows to cross-compare predominant classes of lipids present in eukaryotic species. Each large circle contains lipid types specifically enriched in the respective organism, while intersections contain common types of lipids. Note that in the case of human and fly, relative abundance of phosphatidylethanolamines (PE) and phosphatidylcholines (PC) is inverse, which is indicated by the " $>$ " symbol. Importantly, color coding was used to indicate which tissue or cellular compartments the lipid category in question belongs, while shapes represent different classes of lipids, as explained in the bottom left. Names of lipid types abbreviated as follows: Triacylglycerols (TAG), Diacylglycerols (DAG), Phosphatidylethanolamines (PE), Phosphatidylcholines (PC), Phosphatidylinositols (PI), Phosphatidylserines (PS), Phosphatidylglycerols (PG), Ether lipids (PE-O and PC-O), Phosphorylethanolamine ceramides (CerPE), Hexosyl ceramides (Hex-Cer), Hydroxylated sphingolipids (OH-Sph), Ergosteryl Ester (ErgEst), Ergosterol (ES), sphingomyelin (SM), Sterol (St)

been found to be both unique and highly conserved across evolution, in such a way that only the plasma membrane is distinct in its lipid composition between yeast and man. However, various laboratories trying to determine the lipid composition of the nuclear envelope, even within the same organism, obtained inconsistent results; hence the precise lipid composition of the nuclear envelope remains uncertain. This likely reflects several unique aspects of the nucleus as a membrane bound compartment. First, the nucleus has two distinct lipid bilayers, the inner and outer nuclear membranes, each of which could have a distinct composition. The outer nuclear membrane is physically continuous with the ER, and so ER lipid species can freely diffuse and mix with it.

Nonetheless, proteomics has identified a number of lipid synthesis enzymes (e.g., phospholipid synthetases, phospholipid hydrolases, transferases of fatty acids, PL/lysophospholipids, sphingolipids, etc.) in the inner nuclear membrane that could be responsible for generating its distinct composition [25]. These enzymes represent an important functional principle to regulate precursor/product and mediator lipidomic effects in epigenetic control. Furthermore, the minor lipid species with the shortest half-life are the most important and potent ones (Prof. Gerd Schmidt, personal communication).

Second, the nuclear envelope often has invaginations that contain mitochondria, perhaps to swiftly provide energy for nuclear functions, whose numbers vary enormously. Unfortunately, they are almost impossible to extract while maintaining the nuclear membrane intact. Finally, the nucleus has its own storage of molecules, such as cholesterol and inositol phosphate species, which makes it impossible to determine if 
Table 1: GO-terms linked to lipids for proteins found in the nuclear envelope (NE) by proteomics and their enrichment compared to all GO-term-linked proteins encoded by the genome.

\begin{tabular}{|c|c|c|c|c|}
\hline GO-term & $\begin{array}{c}\mathrm{NE} \\
\text { proteins }\end{array}$ & $\begin{array}{c}\text { Percentage } \\
\text { of NE } \\
\text { protein }\end{array}$ & $\begin{array}{l}\text { Percentage } \\
\text { of all in } \\
\text { genome }\end{array}$ & P-value \\
\hline Membrane organization & 173 & 7.3 & 3.0 & $5.18 e-23$ \\
\hline Nucleus organization & 40 & 1.7 & 0.3 & $1.61 \mathrm{e}-16$ \\
\hline $\begin{array}{l}\text { Endomembrane system } \\
\text { organization }\end{array}$ & 91 & 3.9 & 1.4 & $1.78 \mathrm{e}-15$ \\
\hline Localization within membrane & 14 & 0.6 & 0.1 & $1.75 e-03$ \\
\hline $\begin{array}{l}\text { Response to topologically } \\
\text { incorrect protein }\end{array}$ & 42 & 1.8 & 0.4 & $7.76 e-12$ \\
\hline Lipid metabolic process & 172 & 7.3 & 4.0 & $4.27 e-11$ \\
\hline Neutral lipid metabolic process & 31 & 1.3 & 0.3 & $1.02 \mathrm{e}-07$ \\
\hline Membrane disassembly & 19 & 0.8 & 0.1 & $4.92 \mathrm{e}-10$ \\
\hline $\begin{array}{l}\text { Establishment of protein } \\
\text { localization to membrane }\end{array}$ & 59 & 2.5 & 0.9 & $8.34 \mathrm{e}-10$ \\
\hline Lipid catabolic process & 41 & 1.7 & 0.7 & $2.39 e-03$ \\
\hline Glycero-lipid metabolic process & 55 & 2.3 & 1.1 & $2.94 \mathrm{e}-03$ \\
\hline $\begin{array}{l}\text { Arachidonic acid metabolic } \\
\text { process }\end{array}$ & 17 & 0.7 & 0.2 & $9.26 e-03$ \\
\hline $\begin{array}{l}\text { Fatty acid metabolic } \\
\text { process }\end{array}$ & 62 & 2.6 & 0.9 & $2.50 e-09$ \\
\hline $\begin{array}{l}\text { Long-chain fatty acid metabolic } \\
\text { process }\end{array}$ & 24 & 1.0 & 0.3 & $3.4 \mathrm{e}-04$ \\
\hline $\begin{array}{l}\text { Very long chain fatty acid } \\
\text { metabolic process }\end{array}$ & 10 & 0.4 & 0.1 & $4.70 e-03$ \\
\hline Triglyceride metabolic process & 29 & 1.2 & 0.3 & $5.08 \mathrm{e}-07$ \\
\hline $\begin{array}{l}\text { Triglyceride biosynthetic } \\
\text { process }\end{array}$ & 17 & 0.7 & 0.2 & $2.8 e-04$ \\
\hline Fatty acid biosynthetic process & 28 & 1.2 & 0.4 & $7.4 e-04$ \\
\hline Lipid biosynthetic process & 90 & 3.8 & 1.7 & $8.47 e-09$ \\
\hline $\begin{array}{l}\text { Neutral lipid biosynthetic } \\
\text { process }\end{array}$ & 17 & 0.7 & 0.2 & $4.4 \mathrm{e}-04$ \\
\hline $\begin{array}{l}\text { Carbohydrate biosynthetic } \\
\text { process }\end{array}$ & 38 & 1.6 & 0.6 & $3.37 e-05$ \\
\hline $\begin{array}{l}\text { Internal protein amino acid } \\
\text { acetylation }\end{array}$ & 33 & 1.4 & 0.4 & $3.89 e-06$ \\
\hline
\end{tabular}

To see original data created using Termfinder [29] from which Table was generated you can visit: https://github.com/AlastairKerr/Zhdanov-Kagansky.

the isolated cholesterol species is intra-nuclear or membraneassociated. Though the precise lipid composition of the nuclear envelope remains elusive, the protein composition of the nuclear envelope has been comprehensively determined [25-28]. Many proteins involved in lipid synthesis and membrane function were identified based on GO-term associations.

It was found that certain types of lipid-related proteins and enzymes were particularly enriched in the nuclear envelopes, compared to their percentage representation against all GOterm-associated proteins encoded in the genome (Table 1). The identification of so many proteins involved in lipid biogenesis argues that during interphase, where nuclear size increases two-threefold in S-phase, new lipid species are generated inside the nucleus, and not from lateral diffusion from the ER. This further supports the possibility that the lipid composition of the inner nuclear membrane could differ from that of the outer nuclear membrane/ER. Such lipid synthesizing and metabolic proteins in the nuclear envelope could also function to support a myriad of possible lipid modifications on chromatin and their potential roles in epigenetic regulation.

Hypothesis: Proteins involved in lipid metabolism are strikingly enriched within the nuclear envelope, which could accommodate dynamic changes in the structure of the nucleus throughout the cell cycle without the need to transport lipids through the hydrophilic cytoplasm and this could also support nuclear lipid signaling and regulatory functions.

The degree to which chromatin could come into contact with the nuclear membrane itself is unclear. Electron microscopy shows electron dense material directly apposed to the membrane, but cannot clarify whether the chromatin loops through the lamin polymer were able to directly interact. Certain nuclear envelope transmembrane (NET) proteins [30] interact directly with chromatin, but whether NETs penetrate beyond the lamin polymer for this interaction or the chromatin penetrates the lamin polymer to reach the NET is yet unclear. Lipid species may also be able to interact with chromatin during mitosis under two circumstances: i) breaking down of nuclear envelope, and ii) reformation of nuclear envelope, when presumably vesicles and tubules containing NETs and associated with chromatin binding proteins assemble around the mitotic chromosomes. Whether the chromatin can directly access the membrane or synthesized lipid species are able to covalently attach to chromatin proteins independent of the membrane, lipid interactions could contribute to compact packaging and silencing of chromatin the nuclear periphery, providing a convenient supplement to the epigenetic control of multi-megabase satellite repeats that account for almost half of the genome in humans [31]. The seemingly surprising fact that lipid synthesizing, modifying, and catabolic enzymes have not yet been identified by functional genomics may be explained by most lipid species being indispensable for the cells for their other signaling and structural roles.

\section{LIPID MODIFICATIONS OF NUCLEAR ENVELOPE PROTEINS}

Many small GTPases that function at the plasma membrane are anchored to the membrane via a covalently added lipid moiety, typically farnesylation, or geranylgeranylation [32]. Such proteins are moderately abundant but sporadic at the plasma membrane and are primarily involved in signaling pathways. In the nuclear envelope, the intermediate filament lamins are post-translationally farnesylated. In contrast to plasma membrane GTPases, the lamins are one of the most abundant proteins in the nucleus, estimated at up to 9 million copies in a single nucleus [33], and assemble into a polymer lining the inner nuclear membrane. There are three genes encoding lamins (LMNA, LMNB1, LMNB2) and each of them has multiple splice variants. The major forms of all three are farnesylated; however, whereas the farnesylation is permanent for lamins B1 and B2, it is only transient for lamin A [32]. Some studies suggest that the different lamin subtypes form distinct networks that layer 
with the permanently farnesylated lamins B1 and B2, more proximal to the membrane than lamin A [34, 35]. Accordingly, mutation of each yields strikingly distinct phenotypes [36]. The most intriguing diseases linked to lamins include, restrictive dermopathy, and the premature ageing progeroid syndromes including Atypical Werner's Syndrome and Hutchison-Gilford Progeria Syndrome (HGPS) [37, 38]. Restrictive dermopathy is embryonic lethal, while the progeroid syndromes are principally characterized by growth retardation, alopecia, facial hypoplasia, premature arteriosclerosis, and osteoporosis. Both syndromes involve a disruption of the normal processing of the lamin A farnesylation. Usually, the farnesyl group is added by a farnesyltransferase to the cysteine within a CaaX motif at the C-terminus of the protein. Next, the last three amino acids are cleaved and the cystein is carboxymethylated. Finally, the last 15 amino acids (so 18 in total) are cleaved, thus releasing the farnesyl group from the processed lamin protein. In the case of restrictive dermopathy, the defect is caused just by the inability to properly remove the $\mathrm{C}$ terminal amino acids along with the farnesyl group. Accordingly, it can also be caused by mutations in the Zmpste24 protease that normally performs the cleavage step. In the case of HGPS, the most common lamin A mutation affects a splice junction so that the exon containing the cleavage site is lost. This results in a permanently farnesylated lamin A protein-like restrictive dermopathy, but it also results in a protein lacking 50 amino acids from the missing exon; so it is more difficult to pin the pathologies on just the lipid moiety.

Nonetheless, the farnesyl group has been a principal target of both disorders and treatment with farnesyltransferase inhibitors proven to be very promising in early in vitro studies [39]; however, effects were minimal when trialed in patients [40]. Table 2 gives the list of lipids involved in cholesterol synthesis and their putative epigenetic roles.

Little is known about whether there are other lipid-modified proteins associated with the inner nuclear membrane or how the presence or absence of the lipid moiety affects lamin polymer assembly as the detailed structure of the lamin polymer itself remains unclear. However, there are not likely to be many other farnesylated proteins, as the CaaX motif is present on few other proteins in the nuclear envelope proteome. Nonetheless, inositol triphosphate signaling has been clearly found in the nuclear envelope [41,42] and probably many more classes of lipid signaling species will be found when the nuclear envelope lipidome is finally directly investigated.

\section{LIPID SPECIES FOUND INSIDE THE NUCLEOPLASM}

Varying lipid classes' content of the nuclei from different cell types was reported almost a century ago [43]. In this study, a relatively high content of lipid classes, especially PL and cholesterol, was found in nuclei (see Figure 2). The overall concept of epigenetic regulation in metazoans, proposed a few years later, was successfully then used to explain drastic phenotypic diversification in development between cells in different tissues as well as in disease [44]. The proteins identified in the nuclear envelope, many of which are highly tissue-specific [25-28], raise the possibility of differences in lipid composition of the inner nuclear membrane between tissues. Such differences, if confirmed experimentally in the nuclear envelope lipidome, could be important for epigenetic control as few studies have found variation in the composition of lipid species associated with chromatin isolated from different cells. For example, compared to the active chromatin from other cell types, the silent chromatin from sperm or avian erythrocytes contained lower amounts of DNA-tightly bound neutral lipid species (e.g. cholesterol) and some PL ones (e.g. CL), but it was mainly composed of PE species $[45,46]$. Lipid components of chromatin in rat liver were reported to be dependent on the cell cycle [47].

Interesting possibilities arise from recent discoveries suggesting that lipid species forming distinct microdomains can interact with subnuclear structures [48]. Electron microscopy analysis showed that SM species is present in nuclear domains active in DNA replication, transcription, and possibly in different steps of mRNA processing as well [49]. On this basis, it was proposed that nuclear lipid microdomains might serve as a platform for transcription. These were suggested to be the anchoring point of the active chromatin in liver cells [50]. In a recent study of normal and cancer cells from liver, the authors found differences not only in the lipid composition of the nuclear lipid rafts and lipid microdomains, but also in the proteins involved in carcinogenesis associated with these structures, such as STAT3, Raf1, and PKC $\zeta$ [51]. Interesting additional possibilities are offered by covalent lipid modifications of proteins or lipidization, which will be further reviewed.

\section{LIPID SPECIES IN DIRECT ASSOCIATION WITH DNA AND CHROMATIN}

As early as the 1950s, lipid species was observed in complex with nucleohistones prepared from calf thymus [52]. In that report, the authors also state that the same lipid species was sometimes co-purified with isolated histones, but not with the DNA alone, suggesting that the lipid species could be bound to histones. SM species was proposed as a possible candidate based on chemical evidence from previous studies [53, 54]. A possible function for such modifications may be suggested by a few studies showing that acidic PL species (including CL, PS, PI, and PG species) promote more open transcriptionally permissive chromatin structures. In contrast, neutral lipids (PC, PE, and SM species) appear to act in the opposite way, condensing chromatin fibers $[19,55]$. Thus, lipid modification of histones could contribute similarly to epigenetic marks such as histone acetylation and methylation. Nonetheless, it also appears that lipid species can directly bind to DNA as well. In vitro studies with synthetic DNA polynucleotides reveal that neutral lipids (oleic acid and cholesterol species) can be bound tightly to the DNA double helix [56] but not to triplex DNA. Spectroscopic results indicate that oleic acid molecule shows molecular recognition of AT motifs by minor groove binding. In silico modeling suggests that PL species binding to the minor groove is energetically favorable, with input from hydrogen, van der 

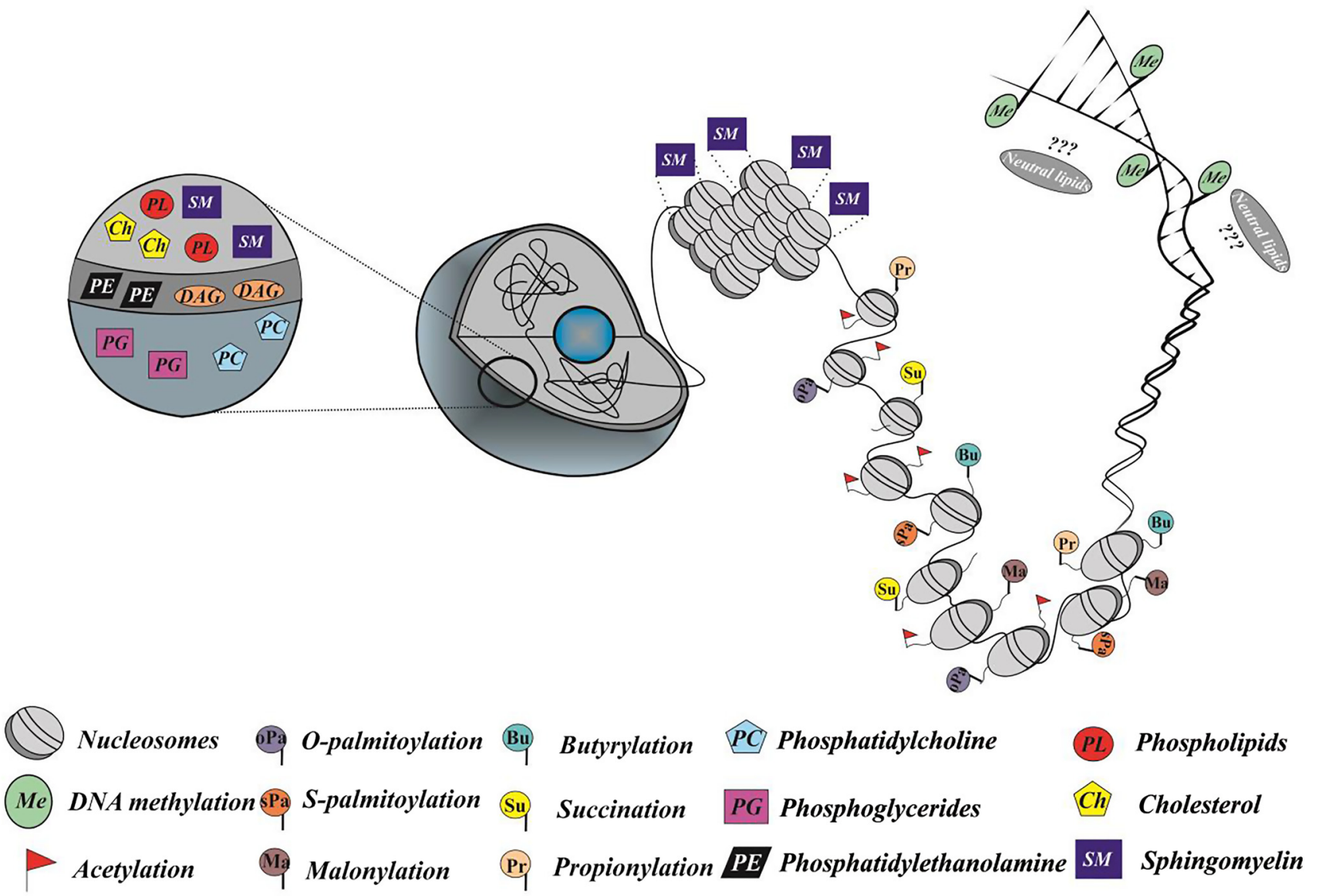

\section{(DAG Diacylglycerol}

Figure 2. Functional distribution of nuclear lipids relative to chromatin. Schematic of the eukaryotic nucleus (center) and major lipids found in the nucleoplasm and nuclear membrane (left), with progressive zoom-in of the chromatin fibers (right). Coded representations of various lipid moieties found to modify core histones (explained in Table 2) are given as circles with abbreviations, key in the bottom left. Sphingomyelin (SM) is proposed to interact with chromatin, as it was originally co-purified with chromatin, while indication of hypothetical interactions of neutral lipids with DNA is only supported by findings in bacteria and computer simulations and is not based on experimental data in eukaryotes. Lipid types and lipid chromatin modifications are listed below the cartoon.

Waals, and hydrophobic bonds [56-58]. Since there is a lipid fraction in the nucleus, apparently tightly bound to DNA, the possibility of a new informational level in genomic DNA ("lipid code") was postulated: lipids might specifically bind to the DNA in a sequence-specific way, and have significant functional roles for genome regulation. We will next discuss ways to understand this information, and what are the putative functions of the genome regulation via lipid species.

\section{LIPID MODIFICATIONS OF CHROMATIN PROTEINS}

Covalent post-translational modifications greatly expand the functional protein repertoire of cells, allowing dynamic regulation of protein function, level of activity, localization, binding partners, and many more [59]. Acylation of lysine residues represents a notable class of modifications. The most famous modification of this class, acetylation, was discovered on histone tails. Acetylation would change histones' charge and consequently their binding partners [60]. Since then, it has become clear that most proteins in eukaryotes are acetylated [61]. Lysine propionylation and butyrylation were also found on yeast histones less than a decade ago $[62,63]$, and their addition to lysines was predicted to promote significant hydrophobicity to the proteins with important implications for their structure and function (see Figure 2). Furthermore, lysine malonylation and succination were discovered very recently by the combination of mass spectrometry and bioinformatics [64]. Succinyl-CoA and malonyl-CoA are important metabolites in the tricarboxylic acid cycle and fatty acid biosynthesis, and therefore the effect of their coupling to chromatin might be twofold: changing the properties of chromatin and removal of these moieties from the metabolic pool [65]. It has proven to be problematic to identify long-chain fatty acid species as covalent attachments on proteins, presumably due to methodological complications such as the use of polar solvents in chromatin isolation methods, which prevents the enrichment of hydrophobic 
Table 2: Lipid modifications of histone proteins from components of cholesterol synthesis pathway and their putative roles.

\section{Other possible histone modifications \\ (Cholesterol synthesis Pathway)}
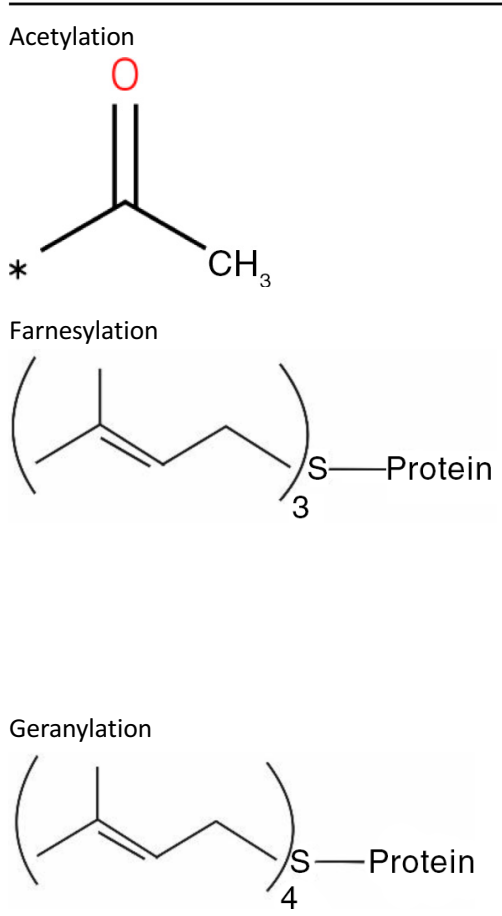

Mevalonate<smiles>O=C(O)CC(O)CCO</smiles>

Dolichols

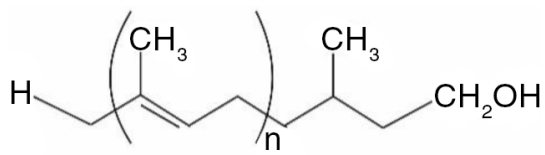

Putative roles

- Alteration of charge distribution by conversion ofpotentially cationic side chains into groups. Change of binding partners.

- Possible regulation of the recruitment of initiating transcription factors.For instance, ubiquitylation is blocked on the Lys side chains from, thus prolonging the half-life of the p53 protein molecule.

- Lamin proteins are generally farnesylated to increase the hydrophobicity of the proteins so that the final mature protein can be targeted to the nuclear envelope.

- Lamin B are permanently farnesylated, whilelamin A undergoes a preprocessing step, where the farnesyl group is generally cleaved.

- Progerin, or farnesylated lamin accumulation causes changes in the levels of heterochromatin and irregular telomere lengths, thus affecting gene expression, however these effects are pharmacologically reversible.

- Progerin may also affect gene regulation by altering the levels or localization of remodeling and modifying factors.

- It has also been observed that inhibition offarnesyl cleavage results in HGPS and affects nuclear morphology and cell proliferation [4].

- Insulin is observed to activate geranylgeranyltransferase (GGT) and that further increases geranylgeranylated Rho-A [1].

- Absence of geranylgeranylated form of Rho-A has been observed to reduce HDAC1 expression and degradation of vitamin D [3].

- Inhibition of GGT results in increased insulin sensitivity of c-fos and Pip92 genes, which areprotooncogene and growth-factor induced gene respectively [1].

- Precursor for mevalonate formation is acetyl-CoA and the rate-limiting step in this pathway is the conversion of HMG-CoA tomevalonate, which can be inhibited by statins.

- Statins can downregulate HDAC2, both directly via competitive inhibition and indirectly via mTOR pathway. They can also reduce DNA methylation via RAS signaling.

- Simvastatin,which can reduce the production of NADPH, has a more emphasized epigeneticrole as this reduction can affect class 1 HDAC activity and the regulation of PARP, thus influencing DNA repair and chromatin dynamics [3].

- These are long chain fatty acids made of repeating numbers of isoprene groups ( $n=17-21)$.

- Dolichol pyrophosphate is the precursor for N-glycosylation of proteins, which occurs by addition of sugar molecules to the former.

- Although a direct role in epigenetics has not bestudied so far, it is possible that they might influence protein biochemistry, as N-glycosylation is one of the post-translational modification enriching protein structure and function.

- Besides, they are produced from Mevalonate, the inhibition of which has serious epigeneticimplications as mentioned above [2]. compounds like lipid species. However, studies by Zou et al. [66] and Wilson et al. [67] provided two different approaches in identifying two long fatty acylations on histones $\mathrm{H} 4$ and $\mathrm{H} 3$. In the former study, lysophosphatidylcholine acyltransferase-I was shown to translocate to the nucleus in the presence of calcium ions, to mediate 0 -palmitoylation of serine 47 on histone $\mathrm{H} 4$ Surprisingly, this modification was found to increase global levels of activated PolII, and therefore positively regulate transcription [66]. In the latter study, chemical reporters were used to detect a range of fatty acid species (myristic, palmitic, and stearic acids) covalently coupled to a large number of nuclear and cytosolic proteins [67]. Interestingly, all of the molecular lipid species mentioned, are starting-, intermediate-, or end-products of the Fatty Acid Synthase (FAS)-complex. Particularly interesting was the finding that histone $\mathrm{H} 3$ variants were S-palmitoylated at cysteine 110, which could affect the ability of H3 to form a tetramer with $\mathrm{H} 4$, which in turn would have radical consequences for the affected chromatin. The attractive possibility is that this modification might change the binding properties of the modified chromatin to the nuclear membrane and nuclear lamins, as their carboxyl-terminal tails interact with H3 [68]. Lipid modifications of histones are summarized in Table 3.

As it can be observed from Table 3, it can be hypothesized that histone acetylation and deacetylation could act as a regulatory 
Table 3: Lipid modifications of histone proteins from components of Fatty Acid Synthase pathway and their putative roles

\begin{tabular}{|c|c|}
\hline $\begin{array}{l}\text { Histone Modifications (FAS } \\
\text { pathway) }\end{array}$ & Putative roles \\
\hline cetylation & $\begin{array}{l}\text { - Alteration of charge distribution by conversion of potentially cationic side chains into groups. Change of } \\
\text { binding partners. } \\
\text { - Possible regulation of the recruitment of initiating transcription factors. For instance, ubiquitylation is blocked } \\
\text { on the Lys side chains from, thusprolonging the half-life of the p53 protein molecule. }\end{array}$ \\
\hline $\begin{array}{r}\text { Propionylation } \\
\qquad \mathrm{O}\end{array}$ & $\begin{array}{l}\text { - Charge neutralization and nonpolar enhancement leads to effective promoting of surface hydrophobicity and } \\
\text { protein aggregation. }\end{array}$ \\
\hline tion & $\begin{array}{l}\text { - Grants hydrophobicity with important implications for histones' structure and function. } \\
\text { - Neutralization of the charge often has a profound impact on the substrate proteins. } \\
\text { - Regulation of tissue-specific cellular metabolic pathways in response to cellular physiological conditions. }\end{array}$ \\
\hline Malonylation & $\begin{array}{l}\text { - Interferes with histone-DNA interaction, causing loss of cell viability. } \\
\text { - Change of a positively charged residue to a negative charge, thus inducing significant structural changes. } \\
\text { - Production of a two-charge shift in the substrate residues. }\end{array}$ \\
\hline Succinylation & $\begin{array}{l}\text { - Unique functional consequences. } \\
\text { - Change of chromatin properties. } \\
\text { - Biological roles related to cellular metabolism: removal of these moieties from the metabolic pool. }\end{array}$ \\
\hline O-palmitoylation & $\begin{array}{l}\text { - Positive regulation of transcription though increase in global levels of PollI. } \\
\text { - Lpcat1-mediated } O \text {-palmitoylation of histone H4: } \\
\text { O Modulation of histone interaction with other scaffolding molecules to alter nuclear signaling events, or } \\
\text { - Involvement in augmentation of epigenetic events that act in concert with other histone modifications. } \\
\text { - Complex biological consequences that may affect fundamental nuclear processes involving gene expression, } \\
\text { cell cycle progression, or mitosis. }\end{array}$ \\
\hline S-palmitoylation & $\begin{array}{l}\text { - Affects tetramer formation of } \mathrm{H} 3 \text { with } \mathrm{H} 4 \text {. } \\
\text { - Change of binding properties of the modified chromatin to the nuclear membrane and nuclear lamins. } \\
\text { - Stabilization of hydrophobic interactions within one or between several nucleosomes, or nucleosomes and } \\
\text { other chromatin proteins. }\end{array}$ \\
\hline
\end{tabular}

principle behind the precursor, intermediate and product epigenetic control as the factors mentioned in the table belong to one of the above three categories in Fatty Acid Synthase (FAS) pathway. Regulation of chromatin function by protein lipidation is not likely to be limited to histones. Palmitoylation of the heterochromatin-associated RAP1-associated protein Rif1 in yeast leads to its relocalization to the nuclear periphery, which increased silencing of the associated peripheral chromatin loci [69]. However, a more recent study indicated that palmitoylation is nonessential for this function of Rif1 [70], suggesting that the nuclear envelope relocalization is sufficiently important that multiple mechanisms have been evolved to target Rif1 to the periphery.

Finally, there are other lipid modifications of proteins, such as geranylgeranylation, phosphatidylethanolamination, glycosyl- phosphatidylation [71], and phosphatidylcholination and phosphatidylserination as important regulators of terminal LC3activation in the control of autophagy and apoptosis [72,73]

With the wide range of lipid modifications already found on chromatin, it would not be surprising if these remaining protein lipid modifications could also be observed at chromatin level, when they are studied in the future. We expect that in the near future, using the combination of proteomic and lipidomic methods of chromatin purification will allow its isolation in the form of the intact "liponucleoprotein", to allow complete classification of lipid and fatty acid species covalently linked to the amino acids. Even more exciting will be the understanding of the functions of these modifications, similarly to incredible variety of simpler moieties such as methylation, which was long considered to be inert (Prof. Colyn Crane-Robinson, personal communication). 


\section{TOWARD UNCOVERING THE ROLES LIPID SPECIES PLAY IN EPIGENETICS: FROM METABOLIC REGULATION OF GENE EXPRESSION TO A METABOLOEPIGENETIC CODE?}

The natural fatty acids butyrate and lactate affect chromatin structure and function leading to the inhibition of histone deacetylase enzymes (HDACs), as well as DNA methylation, thus resulting in global changes to gene expression and chromosome functions $[74,75]$. In a similar context, there have also been reports suggesting a role for HDACs in lipid metabolism. However, lipid and fatty acid biosynthesis seems to be connected with epigenetic regulation more broadly. Levels of acetyl-CoA are critical for the proliferation of eukaryotic cells [76], as it is a source of ATP in the carbon cycle, as well as the source of carbon for the production of lipids, fatty acids, and sterols species. Moreover, it is necessary for histone acetylation in yeast [77] and for tumorigenesis [78]. Co-dependence of both lipid synthesis and histone acetylation on the same pool of acetyl-CoA is illustrated by a few lines of evidence. First, as with most genes, there is regulation of transcription of acetyl-CoA carboxylase, and other metabolic genes by histone acetylation/deacetylation in the promoter regions of these genes [79]. Second, direct protein acetylation of the metabolic proteins, including ones responsible for lipid biosynthesis, is important for their activity [61]. Third, it was recently shown that acetyl-CoA synthetase (ACSS2) is overexpressed in a large proportion of human tumors and is responsible for both lipid synthesis and acetylation of histones [80]. Like acetyl-CoA, 1-carbon metabolism is also crucial for lipid synthesis [80]. S-adenosine methionine (SAM) is a common donor of the methyl group for the processes of methylation of DNA (DNMT1 and DNMT3 in mammals), proteins (such as MLL3, Suv39, and Ezh2), and lipid species (e.g. PE $\mathrm{N}$-methyltransferase [81]).

Concerning S-adenosine methionine (SAM) and DNA-methylation it should be noted that the homocysteine metabolism is critically influenced by choline/betaine, which explains the coincidence of hyperhomocysteinemia and enhanced PC-synthesis under fibrate therapy (choline steeling mechanism) $[82,83]$. The very exciting finding that methionine adenosyltransferase (MAT) is recruited to specific genes to provide SAM locally for usage by the repressive methyltransferases [84] suggests that at least in some cases chromatin modifications can happen using locally produced substrates to avoid competition with the cytosolic pool. This idea resonates with the aforementioned extensive presence of lipid metabolism and biogenesis enzymes within the nuclear membrane. Thus, the concept behind the review may become formulated as the important role of lipid species from both the FAS-pathway and the cholesterol synthesis pathway in epigenetic control. In addition to the maintenance of the membrane itself, this could serve to provide the repertoire of chemical moieties required for the modification of nuclear proteins, including chromatin proteins. It will be intriguing to determine which metabolites contribute to the acetylation and methylation of proteins including histones, as it will have important implications for cancer epigenetics and the targeting of these marks in pharmacological therapies. Further experiments in the emerging field of metaboloepigenetics are warranted based on recent findings, and should be possible due to recent developments in metabolomics, proteomics, and transcriptomics. Another dimension for lipid control over gene regulation has recently opened up due to the identification of the covalent "lipidation" of chromatin proteins discussed earlier. An attractive possibility is that locus-specific lipidation of chromatin allows hydrophobic interactions with specific regions of the inner nuclear membrane and nuclear lipid rafts, and therefore functional control through nuclear compartmentalization and packaging together with other lipidated chromatin domains, and/or temporal changes in chromosome territories.

In addition, there are miRNAs, which play a role in lipogenesis. For example, miR-33 (miR-33a and miR-33b), transcribed along with SREBF gene family controls lipid metabolism and fatty acid oxidation. miR-33 null mice were observed to have high levels of HDL cholesterol. On the other hand, the targets of miR-33* are diverse, inhibiting genes like NPC1, CROT, IRS2 and SRC family. Each of these genes have variety of roles from insulin signaling to cholesterol efflux. Since the discovery of miR-33 family, there are raising evidences suggesting the role of miRNAs in lipid biology [85,86]. Similarly, miR-122 is a potent regulator of cholesterol metabolism. It has been observed that inhibition of miR-122 reduces the levels of cholesterol and triglyceride. Likewise, miR-27a/b, miR-34a, miR-378/378* and miR-21 have approximately 14 gene targets put together, which target different aspects of lipid metabolism and homeostasis [86].

If the direct modification of DNA by lipids is subsequently shown in eukaryotes, a similar regulation by DNA-membrane interactions would be possible. While we still need more extensive data on direct interaction between DNA and/or chromatin with certain lipid species to properly model the roles of these interactions, it is an attractive hypothesis that, as the nuclear lipids surround chromatin, they affect its condensation, mobility, and accessibility, making it more or less transcriptionally permissive, thus epigenetically regulating gene expression and other processes involving chromatin. Since the concept of epigenetics and the histone code was introduced [87], many more histone modifications have been found, and it is currently not clear where chromatin modifications are the real regulators of the chromosome transactions and where they are simply signposts indicating an ongoing process due to other factors they recruit. Although adding lipid modifications to this already extremely complex gene regulation picture further increases its complexity, it may also be the only way we can hope to eventually understand how cell fates are chosen in normal development and how they are disrupted in many human diseases. 


\section{ACKNOWLEDGEMENTS}

This research was made possible due to the support from: MinObrNauki RF (№ 14-80 2014-2015 to RZ, Russia) and A v. Humboldt foundation (to RZ, Russia), Chancellor's Fellowship from University of Edinburgh (to AK and GRB), Erasmus+ grant from Agricultural University of Athens to EM, Erasmus grant from University of Helsinki for AVV and Wellcome Trust grant 095209 to ECS and 092076 from the Wellcome Trust Centre for Cell Biology. The authors thank Colyn CraneRobinson, Nick Gilbert, Jim Allan, Wendy Bickmore, and Nick Hastie for stimulating discussions.

\section{REFERENCES}

[1] Walter A, Margolis D, Mohan R, Blumenthal R. Apocytochrome c induces pH-dependent vesicle fusion. Membr Biochem. 1986; 6(3):217-37.

[2] Taylor DR, Hooper NM. Role of lipid rafts in the processing of the pathogenic prion and Alzheimer's amyloid- $\beta$ proteins. Semin Cell Dev Biol. 2007;18(5):638-48.

[3] Kagan VE, Tyurin VA, Jiang J, Tyurina YY, Ritov VB, Amoscato AA, Osipov AN, Belikova NA, Kapralov AA, Kini V, et al. Cytochrome c acts as a cardiolipin oxygenase required for release of proapoptotic factors. Nat Chem Biol. 2005;1(4):223-32. doi:10.1038/ nchembio727

[4] Forrester JS, Milne SB, Ivanova PT, Brown HA. Computational lipidomics: a multiplexed analysis of dynamic changes in membrane lipid composition during signal transduction. Mol Pharmacol. 2004;65(4):813-21. doi:10.1124/mol.65.4.813

[5] Das S, Dixon JE, Cho W. Membrane-binding and activation mechanism of PTEN. Proc Natl Acad Sci U S A. 2003;100(13): 7491-6. doi:10.1073/pnas.0932835100

[6] Escribá PV, González-Ros JM, Goñi FM, Kinnunen PKJ, Vigh L, Sánchez-Magraner L, Fernández AM, Busquets X, Horváth I, Barceló-Coblijn G. Membranes: a meeting point for lipids, proteins and therapies. J Cell Mol Med. 2008;12(3):829-75.

[7] Allen EE, Facciotti D, Bartlett DH. Monounsaturated but not polyunsaturated fatty acids are required for growth of the deepsea bacterium Photobacterium profundum SS9 at high pressure and low temperature. Appl Environ Microbiol. 1999;65(4): 1710-20.

[8] Jones AL, Hann AC, Harwood JL, Lloyd D. Temperature-induced membrane-lipid adaptation in Acanthamoeba castellanii. Bio-chem J. 1993;290(Pt 1):273-8. doi:10.1042/bj2900273

[9] Carvalho M, Sampaio JL, Palm W, Brankatschk M, Eaton S, Shevchenko A. Effects of diet and development on the Drosophila lipidome. Mol Syst Biol. 2012;8(600):1-17.

[10] Ejsing CS, Sampaio JL, Surendranath V, Duchoslav E, Ekroos K, Klemm RW, Simons K, Shevchenko A. Global analysis of the yeast lipidome by quantitative shotgun mass spectrometry. Proc Natl Acad Sci U S A. 2009;106(7):2136-41. doi:10.1073/pnas. 0811700106

[11] Schuhmann K, Almeida R, Baumert M, Herzog R, Bornstein SR, Shevchenko A. Shotgun Lipidomics on a LTQ Orbitrap mass spectrometer by successive switching between acquisition polarity modes. J Mass Spectrom. 2012;47(1):96-104. doi: $10.1002 / j m s .2031$

[12] Gerl MJ, Sampaio JL, Urban S, Kalvodova L, Verbavatz J-M, Binnington B, Lindemann D, Lingwood CA, Shevchenko A, Schroeder C et al. Quantitative analysis of the lipidomes of the influenza virus envelope and MDCK cell apical membrane. J Cell Biol. 2012;196(2):213-21.
[13] Kalvodova L, Sampaio JL, Cordo S, Ejsing CS, Shevchenko A, Simons K. The lipidomes of vesicular stomatitis virus, semliki forest virus, and the host plasma membrane analyzed by quantitative shotgun mass spectrometry. J Virol. 2009;83(16): 7996-8003. doi:10.1128/JVI.00635-09

[14] Sampaio JL, Gerl MJ, Klose C, Ejsing CS, Beug H, Simons K, Shevchenko A. Membrane lipidome of an epithelial cell line. Proc Natl Acad Sci U S A. 2011;108(5):1903-7. doi:10.1073/pnas. 1019267108

[15] Van Meer G, de Kroon AIPM. Lipid map of the mammalian cell. J Cell Sci. 2011;124(1):5-8.

[16] Van Meer G, Voelker DR, Feigenson GW. Membrane lipids: where they are and how they behave. Nat Rev Mol Cell Biol. 2008;9(2): 112-24.

[17] Struchkov VA, Strazhevskaya NB, Zhdanov RI. Specific natural DNA-bound lipids in post-genome era. The lipid conception of chromatin organization. Bioelectrochemistry. 2002;56(1-2): 195-8.

[18] Hannun YA. The sphingomyelin cycle and the second messenger function of ceramide. J Biol Chem. 1994;269(5):3125-8.

[19] Benadiba M, Miyake JA, Colquhoun A. Gamma-linolenic acid alters Ku80, E2F1, and bax expression and induces micronucleus formation in C6 glioma cells in vitro. IUBMB Life. 2009; 61(3):244-51. doi:10.1002/iub.154

[20] Maraldi NM, Capitani S, Caramelli E, Cocco L, Barnabei O, Manzoli FA. Conformational changes of nuclear chromatin related to phospholipid induced modifications of the template availability. Adv Enzyme Regul. 1984;22:447-64. doi:10.1016/ 0065-2571(84)90025-6

[21] Kamath-Loeb AS, Balakrishna S, Whittington D, Shen JC, Emond MJ, Okabe T, Masutani C, Hanaoka F, Nishimura S, Loeb LA. Sphingosine, a modulator of human translesion DNA polymerase activity. J Biol Chem. 2014;289(31):21663-72. doi:10.1074/jbc. M114.570242

[22] Mizushima T, Natori S, Sekimizu K. Inhibition of Escherichia coli DNA topoisomerase i activity by phospholipids. Biochem J. 1992;285(Pt 2):503-6. doi:10.1042/bj2850503

[23] Simbulan CMG, Taki T, Tamiya-koizumi K, Suzuki M, Savoysky E, Shoji M, Yoshida S. Sulfate- and sialic acid-containing glycolipids inhibit DNA polymerase alpha activity. Biochim Biophys Acta. 1994;1205(1):68-74. doi:10.1016/0167-4838(94)90093-0

[24] Tamura H, Ikegami Y, Ono K, Sekimizu K, Andoh T. Acidic phospholipids directly inhibit DNA binding of mammalian DNA topoisomerase I. FEBS Lett. 1990;261(1):151-4. doi:10.1016/ 0014-5793(90)80658-6

[25] Schirmer EC, Florens L, Guan T, Yates JR, Gerace L. Nuclear membrane proteins with potential disease links found by subtractive proteomics. Science. 2003;301(5638):1380-2. doi: 10.1126/science.1088176

[26] Korfali N, Wilkie GS, Swanson SK, Srsen V, Batrakou DG, Fairley EAL, Malik P, Zuleger N, Goncharevich A, de las Heras J, et al. The leukocyte nuclear envelope proteome varies with cell activation and contains novel transmembrane proteins that affect genome architecture. Mol Cell Proteomics. 2010;9(12): 2571-85. doi:10.1074/mcp.M110.002915

[27] Korfali N, Wilkie GS, Swanson SK, Srsen V, de las Heras J, Batrakou DG, Malik P, Zuleger N, Kerr ARW, Florens L, et al. The nuclear envelope proteome differs notably between tissues. Nucleus. 2012;3(6):552-64. doi:10.4161/nucl.22257

[28] Wilkie GS, Korfali N, Swanson SK, Malik P, Srsen V, Batrakou DG, de las Heras J, Zuleger N, Kerr ARW, Florens L et al. Several novel nuclear envelope transmembrane proteins identified in skeletal muscle have cytoskeletal associations. Mol Cell Proteomics. 2011;10(1), M110.003129. 
[29] Boyle EI, Weng S, Gollub J, Jin H, Botstein D, Michael J, Sherlock G. GO::TermFinder-open source software for accessing Gene Ontology information and finding significantly enriched Gene Ontology terms associated with a list of genes. Bioinformatics 2004;20(18):3710-15.

[30] Worman HJ, Schirmer EC. Nuclear membrane diversity: underlying tissue-specific pathologies in disease? Curr Opin Cell Biol. 2015;34:101-12.

[31] Britten RJ, Kohne DE. Repeated sequences in DNA. Science. 1968;161(3841):529-40. doi:10.1126/science.161.3841.529

[32] Young SG, Fong LG, Michaelis S. Thematic review series: lipid posttranslational modifications. Prelamin A, Zmpste24, misshapen cell nuclei, and progeria-new evidence suggesting that protein farnesylation could be important for disease pathogenesis. J Lipid Res. 2005;46(12):2531-58.

[33] Schwanhausser B, Busse D, Li N, Dittmar G, Schuchhardt J, Wolf J, Chen W, Selbach M. Global quantification of mammalian gene expression control. Nature. 2011;473(7347):337-42. doi: 10.1038/ nature10098

[34] Goldberg MW, Huttenlauch I, Hutchison CJ, Stick R. Filaments made from A- and B-type lamins differ in structure and organization. J Cell Sci. 2008;121(2):215-25. doi:10.1242/jcs. 022020

[35] Shimi T, Kittisopikul M, Tran J, Goldman AE, Adam SA, Zheng Y, Jaqaman K, Goldman RD. Structural organization of nuclear lamins A, C, B1 and B2 revealed by super-resolution microscopy. Mol Biol Cell. 2015. Epub ahead of print.

[36] Burke B, Stewart CL. The nuclear lamins: flexibility in function. Nat Rev Mol Cell Biol. 2013;14(1):13-24. doi:10.1038/nrm3488

[37] De Sandre-Giovannoli A, Bernard R, Cau P, Navarro C, Amiel J, Boccaccio I, Lyonnet S, Stewart CL, Munnich A, Le Merrer M, et al. Lamin a truncation in Hutchinson-Gilford progeria. Science. 2003; 300(5628):2055.

[38] Eriksson M, Brown WT, Gordon LB, Glynn MW, Singer J, Scott L, Erdos MR, Robbins CM, Moses TY, Berglund P, et al. Recurrent de novo point mutations in lamin A cause Hutchinson-Gilford progeria syndrome. Nature. 2003;423(6937):293-8. doi:10.1038/ nature01629

[39] Young SG, Yang SH, Davies BSJ, Jung HJ, Fong LG. Targeting protein prenylation in progeria. Sci Trans Med. 2013;5(171): $171 \mathrm{ps} 3$.

[40] Gordon LB, Kleinman ME, Miller DT, Neuberg DS, GiobbieHurder A, Gerhard-Herman M, Smoot LB, Gordon CM, Cleveland $\mathrm{R}$, Snyder BD et al. Clinical trial of a farnesyltransferase inhibitor in children with Hutchinson-Gilford progeria syn-drome. Proc Natl Acad Sci U S A. 2012;109(41):16666-71. doi:10.1073/ pnas.1202529109

[41] Kusnier C, Cardenas C, Hidalgo J, Jaimovich E. Single-channel recording of inositol trisphosphate receptor in the isolated nucleus of a muscle cell line. Biol Res. 2006;39(3):541-53. doi:10.4067/S0716-97602006000300015

[42] Smrcka AV. Regulation of phosphatidylinositol-specific phospholipase $\mathrm{C}$ at the nuclear envelope in cardiac myocytes. J Cardiovasc Pharmacol. 2015;65(3):203-10. doi:10.1097/FJC. 0000000000000195

[43] Stoneburg CA. Lipids of the cell nuclei. J Biol Chem. 1939; 129(1): 189-96.

[44] Waddington CH. The epigenotype. Int J Epidemiol. 2012;41(1): 10-13. doi:10.1093/ije/dyr184

[45] Struchkov VA, Strazhevskaia NB. DNA-binding lipids: composition and possible functions. Biokhimiia 1993;58(8):1154-75.

[46] Albi E, Viola-Magni MP. Chromatin-associated sphingomyelin: metabolism in relation to cell function. Cell Biochem Funct. 2003;21(3):211-5. doi:10.1002/cbf.1075
[47] Makishima M. Lipid metabolism and nuclear receptors. Seikagaku 2003;75(5):391-5.

[48] Lichtenberg D, Goñi FM, Heerklotz H. Detergent-resistant membranes should not be identified with membrane rafts. Trends Biochem Sci. 2005;30(8):430-6.

[49] Cascianelli G, Villani M, Tosti M, Marini F, Bartoccini E, Viola Magni M, Albi E. Lipid microdomains in cell nucleus. Mol Biol Cell. 2008;19(12):5289-95. doi:10.1091/mbc.E08-05-0517

[50] Albi E, Lazzarini A, Lazzarini R, Floridi A, Damaskopoulou E, Curcio F, Cataldi S. Nuclear lipid microdomain as place of interaction between sphingomyelin and DNA during liver regeneration. Int J Mol Sci. 2013;14(4):6529-41. doi:10.3390/ ijms14046529

[51] Lazzarini A, Macchiarulo A, Floridi A, Coletti A, Cataldi S, Codini M, Lazzarini R, Bartoccini E, Cascianelli G, Ambesi-Impiombato FS, et al. Very-long-chain fatty acid sphingomyelin in nuclear lipid microdomains of hepatocytes and hepatoma cells: can the exchange from C24:0 to C16:0 affect signal proteins and vitamin D receptor? Mol Biol Cell. 2015;26(13):2418-25.

[52] Wilkins MHF, Zubay G, Wilson HR. X-ray diffraction studies of the molecular structure of nucleohistone and chromosomes. J Mol Biol. 1959;1(2):179-IN10.

[53] Bakay B, Kolb JJ, Toennies G. On the component proteins of calf thymus nucleoprotein. Arch Biochem Biophys. 1955;58(1): 144-68. doi:10.1016/0003-9861(55)90101-6

[54] Chayen J, Gahan PB. Lipid components in nucleohistone. Biochem J. 1958;69(4):49.

[55] Manzoli FA, Capitani S, Mazzotti G, Barnabei O, Maraldi NM. Role of chromatin phospholipids on template availability and ultrastructure of isolated nuclei. Advances in Enzyme Regulation 1982;20:247-62.

[56] Zhdanov RI, Strazhevskaya NB, Jdanov AR, Bischoff G. A spectroscopic and surface plasmon resonance study of oleic acid/DNA complexes. J Biomol Struct Dyn. 2002;20(2):231.

[57] Zhdanov RI, Salafutdinov II, Arslan A, Ibragimova MY. New marker of FAME profile of Pseudomonas aurantiaca total lipids. Dok Biochem Biophy. 2012;445(1):183-6.

[58] Zhdanov RI, Kern D, Lorenz W, Ibragimova MY. Lipid and fatty acid profiles of Pseudomonas aurantiaca DNA-bound lipids deter-mined by mass spectrometry. Microbiology. 2015;84(1): 34-40.

[59] Walsh CT, Garneau-Tsodikova S, Gatto GJ. Protein posttranslational modifications: the chemistry of proteome diversifications. Angew Chem Int Ed Engl. 2005;44(45):7342-72.

[60] Norris KL, Lee JY, Yao TP. Acetylation goes global: the emergence of acetylation biology. Sci Signal. 2009;2(97):pe76. doi:10.1126/scisignal.297pe76

[61] Zhao S, Xu W, Jiang W, Yu W, Lin Y, Zhang T, Yao J, Zhou L, Zeng Y, Li H, et al. Regulation of cellular metabolism by protein lysine acetylation. Science. 2010;327(5968):1000-4.

[62] Chen Y, Sprung R, Tang Y, Ball H, Sangras B, Kim SC, Falck JR, Peng J, Gu W, Zhao Y. Lysine propionylation and butyrylation are novel post-translational modifications in histones. Mol Cell Proteomics. 2007;6(5):812-9. doi:10.1074/mcp. M700021-MCP200

[63] Zhang K, Chen Y, Zhang Z, Zhao Y. Identification and verification of lysine propionylation and butyrylation in yeast core histones using PTMap software. J Proteome Res. 2009;8(2):900-6.

[64] Xie Z, Dai J, Dai L, Tan M, Cheng Z, Wu Y, Boeke JD, Zhao Y. Lysine succinylation and lysine malonylation in histones. Mol Cell Proteomics. 2012;11(5):100-7.

[65] Lee S. Post-translational modification of proteins in toxicological research: focus on lysine acylation. Toxicol Res. 2013;29(2): 81-6. doi:10.5487/TR.2013.29.2.081 
[66] Zou C, Ellis BM, Smith RM, Chen BB, Zhao Y, Mallampalli RK. Acyl-CoA:lysophosphatidylcholine acyltransferase I (Lpcat1) catalyzes histone protein 0-palmitoylation to regulate mRNA synthesis. J Biol Chem. 2011;286(32):28019-25.

[67] Wilson JP, Raghavan AS, Yang YY, Charron G, Hang HC. Proteomic analysis of fatty-acylated proteins in mammalian cells with chemical reporters reveals S-acylation of histone H3 variants. Mol Cell Proteomics. 2011;10(3):M110.001198.

[68] Hideo T, Charles G, Larry G. A chromatin binding site in the tail domain of nuclear lamins that interacts with core histones. J Cell Biol. 1995;131(1):33-44. doi:10.1083/jcb.131.1.33

[69] Park S, Patterson EE, Cobb J, Audhya A, Gartenberg MR, Fox CA. Palmitoylation controls the dynamics of budding-yeast heterochromatin via the telomere-binding protein Rif1. Proc Natl Acad Sci U S A. 2011;108(35):14572-7. doi:10.1073/pnas.1105 262108

[70] Peace JM, Ter-Zakarian A, Aparicio OM. Rif1 regulates initiation timing of late replication origins throughout the $S$. cerevisiae genome. PLoS One. 2014;9(5):e98501. doi:10.1371/journal. pone. 0098501.s006

[71] Resh MD. Covalent lipid modifications of proteins. Curr Biol. 2013;23(10):R431-5. doi:10.1016/j.cub.2013.04.024

[72] Birmingham CL, Canadien V, Gouin E, Troy EB, Yoshimori T, Cossart P, Higgins DE, Brumell JH. Listeria monocytogenes evades killing by autophagy during colonization of host cells. Autophagy. 2007;3(5):442-451.

[73] Meng X, Wang M, Sun G, Ye J, Zhou Y, Dong X, Wang T, Lu S, Sun $\mathrm{X}$. Attenuation of A 325 -35-induced parallel autophagic and apoptotic cell death by gypenoside XVII through the estrogen receptor-dependent activation of Nrf2/ARE pathways. Toxicol Appl Pharmacol. 2014;279(1): 63-75. doi:10.1016/j.taap.2014. 03.026

[74] Davie JR. Inhibition of histone deacetylase activity by butyrate. J Nutr. 2003;133(7): 2485S-93S.

[75] Latham T, MacKay L, Sproul D, Karim M, Culley J, Harrison DJ, Hayward L, Langridge-Smith P, Gilbert N, Ramsahoye BH. Lactate, a product of glycolytic metabolism, inhibits histone deacetylase activity and promotes changes in gene expression. Nucleic Acids Res. 2012;40(11):4794-803. doi:10.1093/nar/ gks066

[76] Shi L, Tu BP. Acetyl-CoA induces transcription of the key G1 cyclin CLN3 to promote entry into the cell division cycle in Saccharomyces cerevisiae. Proc Natl Acad Sci. 2013;110(18): 7318-23. doi:10.1073/pnas.1302490110

[77] Cai L, Sutter BM, Li B, Tu BP. Acetyl-CoA induces cell growth and proliferation by promoting the acetylation of histones at growth genes. Mol Cell. 2011;42(4):426-37. doi:10.1016/j.molcel. 2011. 05.004

[78] Comerford SA, Huang Z, Du X, Wang Y, Cai L, Witkiewicz AK, Walters H, Tantawy MN, Fu A, Manning HC, et al. Acetate dependence of tumors. Cell. 2015;159(7):1591-602. doi:10. 1016/j.cell.2014.11.020

[79] Kristjuhan A, Walker J, Suka N, Grunstein M, Roberts D, Cairns BR, Svejstrup JQ. Transcriptional inhibition of genes with severe histone $\mathrm{H} 3$ hypoacetylation in the coding region. Mol Cell. 2002;10(4):925-33. doi:10.1016/S1097 -2765(02)00647-0

[80] Locasale JW. Serine, glycine and the one-carbon cycle: cancer metabolism in full circle. Nat Rev Cancer. 2013;13(8):572-83. doi:10.1038/nrc3557

[81] Kaneshiro T, Law JH. Phosphatidylcholine synthesis in agrobacterium tumefaciens: I. Purification and properties of a phosphatidylethanolamine N-methyltransferase. J Biol Chem. 1964; 239(6):1705-13.

[82] Muskiet FA. The importance of (early) folate status to primary and secondary coronary artery disease prevention. Reprod Toxicol. 2005;20(3):403-10.

[83] Jadavji NM, Deng L, Malysheva O, Caudill MA, Rozen R. MTHFR deficiency or reduced intake of folate or choline in pregnant mice results in impaired short-term memory and increased apoptosis in the hippocampus of wild-type offspring. Neuroscience. 2015;300: 1-9. doi:10.1016/j.neuroscience.2015.04.067

[84] Katoh Y, Ikura T, Hoshikawa Y, Tashiro S, Ito T, Ohta M, Kera Y, Noda $\mathrm{T}$, Igarashi $\mathrm{K}$. Methionine adenosyltransferase II serves as a transcriptional corepressor of Maf oncoprotein. Mol Cell. 2011;41(5):554-66. doi:10.1016/j.molcel.2011.02.018

[85] Moore KJ. microRNAs: small regulators with a big impact on lipid metabolism. J Lipid Res. 2013;54(5): 1159-60. doi:10.1194/jlr. E036954

[86] Yang Z, Cappello T, Wang L. Emerging role of microRNAs in lipid metabolism. Acta Pharma Sin B. 2015;5(2): 145-50. doi:10.1016/j.apsb.2015.01.002

[87] Strahl BD, Allis CD. The language of covalent histone modifications. Nature. 2000;403(6765):41-45.

\section{COMPETING INTERESTS}

Authors declare no competing interests.

\section{PUBLISHING NOTES}

(C) 2016 Zhdanov et al. This work has been published open access under Creative Commons Attribution License CC BY 4.0, which permits unrestricted use, distribution, and reproduction in any medium, provided the original work is properly cited. Conditions, terms of use and publishing policy can be found at www.scienceopen.com.

Please note that this article may not have been peer reviewed yet and is under continuous post-publication peer review. For the current reviewing status please click here or scan the QR code on the right.

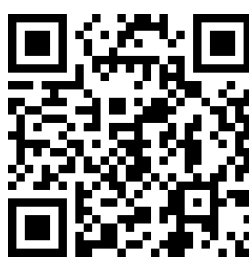

\section{scienceOPEN.com}

research+publishing network 